\title{
OPEN Accurate prediction of terahertz spectra of molecular crystals of fentanyl and its analogs
}

Chun-Hung Wang ${ }^{1}$, Anthony C. Terracciano ${ }^{2,3}$, Artëm E. Masunov ${ }^{1,4,5 \bowtie}$, Mengyu X $\mathrm{u}^{3,6}$ Subith S. Vasu',

Fentanyl is a potent synthetic opioid pain reliever with a high bioavailability that can be used as prescription anesthetic. Rapid identification via non-contact methods of both known and emerging opioid substances in the fentanyl family help identify the substances and enable rapid medical attention. We apply PBEh-3c method to identify vibrational normal modes from 0.01 to $3 \mathrm{THz}$ in solid fentanyl and its selected analogs. The molecular structure of each fentanyl analog and unique arrangement of $\mathrm{H}$-bonds and dispersion interactions significantly change crystal packing and is subsequently reflected in the THz spectrum. Further, the study of THz spectra of a series of stereoisomers shows that small changes in molecular structure results in distinct crystal packing and significantly alters THz spectra as well. We discuss spectral features of synthetic opioids with higher potency than conventional fentanyl such as ohmefentanyl and sufentanil and discover the pattern of THz spectra of fentanyl analogs.

Fentanyl ( $N$-phenyl- $N$-[1-(2-phenylethyl)piperidin-4-yl]propionanilide) ${ }^{1}$ is one kind of synthetic opioid used for pain relief, which has high affinity for $\mu$-opioid receptor and acts as an agonist with higher potency than morphine. Its effective dose $\left(\mathrm{ED}_{95}\right)$ is $0.45-0.60 \mu \mathrm{g} / \mathrm{kg}$, partly due to its $92 \%$ bioavailability ${ }^{2,3}$. A comprehensive review of the history of fentanyl and its analogs has been published in 2018 by Armenian et al. ${ }^{4}$ Carfentanil ${ }^{5}$, sufentanil ${ }^{6}$, alfentanil ${ }^{7}$, and remifentanil ${ }^{8}$ are fentanyl analogs with additional functional groups which enable a higher potency than fentanyl itself.

Once in the body, fentanyl metabolism begins with N-oxidative dealkylation and the products are eventually excreted with the urine $e^{9,10}$. Fentanyl can be administered via inhalation, ingestion, oral exposure, injection, or via transdermal means ${ }^{11,12}$. It is primarily produced in the form of powdered substances, typically white. When obtained outside of a pharmaceutical setting, it may be mixed with heroin and cutting agents or pressed into counterfeit opioid prescription pills ${ }^{13}$. Fentanyl may even be weaponized in the form of aerosol ${ }^{14}$. Widespread use of fentanyl as either a pure compound or mixture with other drugs poses a significant threat for first responders given its ample means of exposure and effective dosing ${ }^{15}$.

Several existing methods may be used for fentanyl and other narcotic detection including: ion mobility spectrometry ${ }^{16,17}$, electron impact mass spectrometry ${ }^{18}$, microtiter plate enzyme analysis ${ }^{19}$, radio immunoassays $^{20}$, thermal desorption direct analysis in real-time mass spectrometry ${ }^{21}$, surface enhanced Raman spectroscopy ${ }^{22,23}$, and differential pulse voltammetry ${ }^{24}$. However, these methods require handling of the substance and/or taking samples of the bodily fluids. Fentanyl analogs have similar structure, but exhibit distinct functional groups, which complicates detection of such synthetic opioids. Additionally, clandestine laboratories do not have stringent protocols for ensuring the proper reaction occurs and novel variations. Thus, desirable means of fentanyl detection must possess the following features: (i) non-contact identification of fentanyl and its derivatives; (ii) accurate detection with minimal false positives and false negatives; (iii) be simple for an operator with no scientific background; and (iv) provide results in seconds.

The method for fentanyl identification which satisfies the above criteria is THz spectroscopy which employs electromagnetic fields in the THz region $(0.1-3.0 \mathrm{THz})$ and is applicable to imaging and sensing ${ }^{25,26}$. Non-polar

\footnotetext{
${ }^{1}$ NanoScience Technology Center, University of Central Florida, 12424 Research Parkway, Orlando, FL 32826, USA. ${ }^{2}$ Department of Mechanical and Aerospace Engineering, University of Central Florida, 4000 Central Florida Blvd, Orlando, FL 32816, USA. ${ }^{3}$ Center for Advanced Turbomachinery and Energy Research, University of Central Florida, 4000 Central Florida Blvd, Orlando, FL 32816, USA. ${ }^{4}$ South Ural State University, Lenin Pr. 76, Chelyabinsk, Russia 454080. 5 National Research Nuclear University MEPhl, Kashirskoye Shosse 31, Moscow, Russia 115409. ${ }^{6}$ Department of Statistics and Data Science, University of Central Florida, 4000 Central Florida Blvd, Orlando, FL 32816, USA. ${ }^{\boxplus}$ email: amasunov@ucf.edu
} 


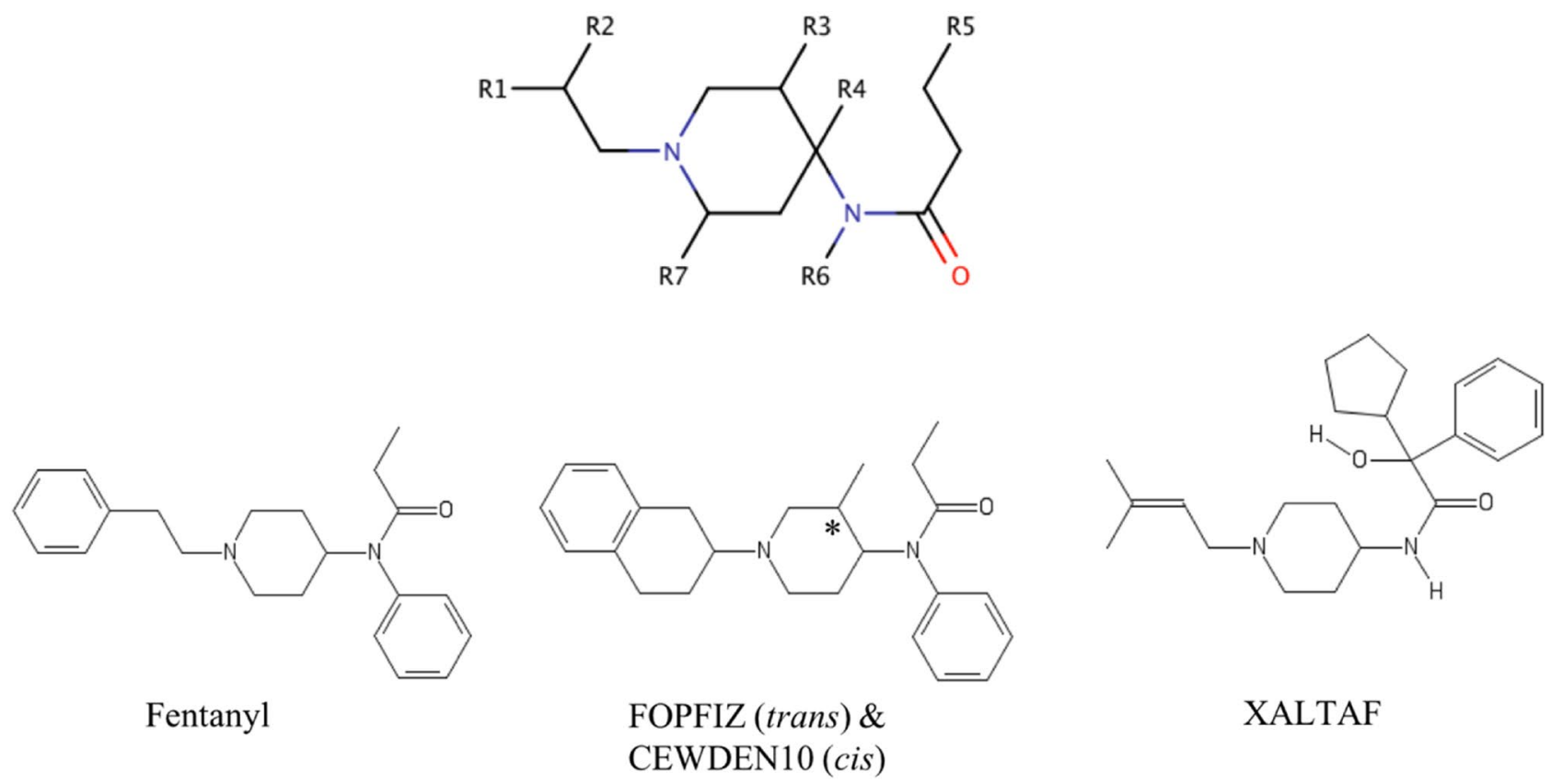

Figure 1. Structure of fentanyl, selected analogs with stereoisomer FOPFIZ/CEWDEN10, and XALTAF; “*” indicates chiral center.

\begin{tabular}{|l|l|l|l|l|l|l|l|}
\hline & R1 & R2 & R3 & $\mathbf{R} 4$ & $\mathbf{R}$ & R6 & R7 \\
\hline Fentanyl & $\mathrm{Ph}$ & $\mathrm{H}$ & $\mathrm{H}$ & $\mathrm{H}$ & $\mathrm{H}$ & $\mathrm{Ph}$ & $\mathrm{H}$ \\
\hline Ohmefentanyl & $\mathrm{Ph}$ & $\mathrm{OH}$ & $\mathrm{Me}$ & $\mathrm{H}$ & $\mathrm{H}$ & $\mathrm{Ph}$ & $\mathrm{H}$ \\
\hline 3-Methylfentanyl & $\mathrm{Ph}$ & $\mathrm{H}$ & $\mathrm{Me}$ & $\mathrm{H}$ & $\mathrm{H}$ & $\mathrm{Ph}$ & $\mathrm{H}$ \\
\hline BIYTAF & $\mathrm{Ph}$ & $\mathrm{OH}$ & $\mathrm{H}$ & $\mathrm{H}$ & $\mathrm{H}$ & $\mathrm{Ph}$ & $\mathrm{H}$ \\
\hline TACDOS/TACDUY & $\mathrm{Ph}$ & $\mathrm{H}$ & $\mathrm{H}$ & $\mathrm{H}$ & $\mathrm{CH}_{2} \mathrm{CO}_{2} \mathrm{H}$ & $\mathrm{Ph}$ & $\mathrm{H}$ \\
\hline FBPIPA & $p$-FPhC(O) $\mathrm{CH}_{2}$ & $\mathrm{H}$ & $\mathrm{H}$ & $\mathrm{H}$ & $\mathrm{H}$ & $\mathrm{Ph}$ & $\mathrm{H}$ \\
\hline BEGZUJ & $\mathrm{Ph}$ & $\mathrm{H}$ & $\mathrm{H}$ & $\mathrm{H}$ & $\mathrm{H}$ & 1-phenyl-3-pyrazolyl & $\mathrm{H}$ \\
\hline JINPAX/VEYCIL & $\mathrm{Ph}$ & $\mathrm{H}$ & $\mathrm{Me}$ & $\mathrm{H}$ & $\mathrm{H}$ & $\mathrm{Ph}$ & $\mathrm{Me}$ \\
\hline IFIGIN & $\mathrm{Ph}$ & $\mathrm{p}$ - $\mathrm{BrPhCO}$ & $\mathrm{Me}$ & $\mathrm{H}$ & $\mathrm{H}$ & $\mathrm{Ph}$ & $\mathrm{H}$ \\
\hline ZIZPON & $\mathrm{Ph}$ & $\mathrm{H}$ & $\mathrm{H}$ & $\mathrm{H}$ & $\mathrm{GeMe}$ & $\mathrm{Ph}$ & $\mathrm{H}$ \\
\hline Carfentanil & $\mathrm{Ph}$ & $\mathrm{H}$ & $\mathrm{H}$ & $\mathrm{CO}_{2} \mathrm{Me}$ & $\mathrm{H}$ & $\mathrm{Ph}$ & $\mathrm{H}$ \\
\hline R-30490 & $\mathrm{Ph}$ & $\mathrm{H}$ & $\mathrm{H}$ & $\mathrm{CH}_{2} \mathrm{OMe}$ & $\mathrm{H}$ & $\mathrm{Ph}$ & $\mathrm{H}$ \\
\hline Sufentanil & 2-thiophenyl & $\mathrm{H}$ & $\mathrm{CH}_{2} \mathrm{OMe}$ & $\mathrm{H}$ & $\mathrm{Ph}$ & $\mathrm{H}$ \\
\hline Alfentanil & 4-ethyl-5-oxo-tetrazol-1-yl & $\mathrm{H}$ & $\mathrm{H}$ & $\mathrm{CH}_{2} \mathrm{OMe}$ & $\mathrm{H}$ & $\mathrm{Ph}$ & $\mathrm{H}$ \\
\hline Thiofentanil & 2-thiophenyl & $\mathrm{H}$ & $\mathrm{H}$ & $\mathrm{CO}_{2} \mathrm{Me}$ & $\mathrm{H}$ & $\mathrm{Ph}$ & $\mathrm{H}$ \\
\hline
\end{tabular}

Table 1. Molecular structures of fentanyl and its analogs.

dielectric objects such as clothes, plastics, paper envelope, and luggage are semi-transparent to $\mathrm{THz}$ radiation minimizing obscuring effects of containers ${ }^{27,28}$. In addition, the narrow range $0.1-3.0 \mathrm{THz}$ of low vibrational frequency between far infrared (IR) and microwave may provide spectroscopic fingerprint, capable to detect fentanyl analogs accurately.

To facilitate THz identification of fentanyl, it is advantageous to assemble a database of fentanyl THz spectra. This can be done, for instance, by quantum mechanical (QM) density-functional theory (DFT) calculations in solid state. Such efforts will enable chemometrics techniques for understanding and validation of reflectance spectroscopy experiment, and the fast prediction techniques by machine learning (ML) methods. In this work, we will establish a library of $\mathrm{THz}$ spectra of fentanyl and its analogs via QM calculation.

Molecule of fentanyl includes three functional moieties: $N$-phenyl-propanamide, piperidine ring, and $N$-phenylethyl ring. Fentanyl analogs we selected for spectral predictions are those with altered pharmacology and toxicology (shown on Fig. 1 and Table 1). Substitution of one methyl group into the piperidine ring in fentanyl results in 3-methylfentany ${ }^{29}$. Substitution of a hydroxyl group between piperidine and phenyl group in 3-methylfentanyl leads to ohmefentanyl ${ }^{30,31}$, which contains the multiple chiral centers denoted by the "^” at particular atom(s). Adding other functional groups to the structure of fentanyl can produce BIYTAF ${ }^{32}(N-(1-(2$-hydroxy2-phenylethyl)-4-piperidyl)- $N$-phenylpropanamide), TACDOS/TACDUY ${ }^{33}$ (5-oxo-5-(phenyl(1-(2-phenylethyl) 
piperidinium-4-yl)amino)pentanoate, JINPAX $(2 R, 5 S)^{34} \& \operatorname{VEYCIL}(2 S, 5 S)^{35}$ (2,5-Dimethyl-1-(2-phenylethyl)4-( $N$-propionylanilino)piperdine), FOPFIZ/CEWDEN10 $0^{36}$ (trans/cis- $N$-(3-Methyl-1-(2-(1,2,3,4-tetrahydro) naphthyl)-piperidin-4-yl)- $N$-phenylpropanamide), FBPIPA $^{37}$ ( $N$-(1-(3-( $p$-fluorobenzoyl)propyl)-4-piperidyl)propionanilide), BEGZUI ${ }^{38}$ ( $N$-(1-(2-Phenylethyl)-4-piperidyl)- $N$-(1-phenyl-3-pyrazolyl)propanamide), IFIGIN ${ }^{39}$ $((1 R)-2-[(3 R, 4 S)-3-$ Methyl-4-( $N$-phenyl- $N$-propionylamino)piperidin-1-yl]-1-phenylethyl $p$-Bromobenzoate), ZIZPON $^{40}$ (1-(2-Phenylethyl)-4-((3-trimethylgermylpropanoyl)phenylamino)piperidinium), or XALTAF ${ }^{41}$ ((2R)-N-(1-(4-Methyl-3-pentenyl)piperidin-4-yl)-2-cyclopentyl-2-hydroxy-2-phenylacetamide).

The presence of the one (FOPFIZ/CEWDEN10), two (JINPAX/VEYCIL), and three (ohmefentanyl) chiral centers results in distinct packing features for crystallized stereoisomers. While it has not been considered here, we also expect hydrates to decrease the intensity of observed absorption peaks as the phonon modes transmitted within the lattice will be damped as water is included ${ }^{42}$. In order to compare with experimental results, 4-methoxymethylfentanyl (R-3049043, $N$-(4-(Methoxymethyl)-1-(2-phenylethyl)-4-piperidinyl)- $N$-phenyl-propanamide), sufentanil $^{6}$, alfentanil ${ }^{7}$, and thiofentanil ${ }^{44}$ are studied computationally as well (see Table 1 ). The stereoisomers have distinct crystal packing and thermodynamic parameters, which determine the difference in properties like solubility and bioavailability.

The detection of fentanyl by THz spectra is possible due to unique phonon properties (molecular motion). Each phonon mode in the given wavelength range corresponds to a hindered molecular rotation (libration), coupled to intramolecular vibrations. Differences in the molecular structure of the fentanyl analogs leads to distinctly different crystalline packing and hence $\mathrm{THz}$ spectra. In general, long-distance intermolecular interaction determines the strength and the position of peaks on THz spectra in the solid state. Specific intermolecular interactions such as hydrogen bonding and $\pi-\pi$ stacking tend to have significant influence of $\mathrm{THz}$ frequency shift ${ }^{45}$.

In order to accurately describe long-distance dispersion interactions for organic molecular solids, we have decided to apply Grimme's PBEh-3c method ${ }^{46}$, based on corrected Perdew-Burke-Ernzerhoff (PBE) ${ }^{47}$ hybrid generalized-gradient-approximation functional. Conformational analyses of fentanyl and its analogs ohmefentanyl $l^{48-50}, 3$-methylfentany ${ }^{49}$, BIYTAF $^{48}$, sufentanil ${ }^{48}$, and alfentanil ${ }^{48,51}$ have been studied previously in different groups by molecular dynamics and molecular docking to understand the docking site of fentanyl analogs to $\mu$-opioid receptor and by DFT calculations to understand stable conformations in gas and aqueous phase. To the best of our knowledge, this is the first time THz spectra of fentanyl and its analogs in solid state had been predicted by DFT calculation. Other factors which may affect measured THz spectra (such as mixture of different fentanyl analogs and hydrates) can be aggressed in our future work by ML methods.

\section{Computational methods}

All the crystal structures of the various fentanyl molecules were obtained from the Cambridge Structural Database ${ }^{52}$. The structures were preprocessed using cif $2 \mathrm{cell}^{53}$ to convert crystallographic information file to the proper input file format. Some of the fentanyl analogs were missing hydrogen atoms, which were added geometrically using Molden program ${ }^{54}$. Marvin ${ }^{55}$ was used for drawing, displaying, and characterizing two-dimensional chemical structures and Mercury ${ }^{56}$ was used for visualizing three-dimensional geometry. Post-processing of the output files to produce the graphical representation of the absorption spectra and vibrational normal modes was done using CRYSPLOT ${ }^{57}$. Microsoft Excel 2019 was used for presenting absorption spectra ${ }^{58}$. CRYSTAL17 $7^{59}$, was utilized for solid-state DFT calculations with periodic boundary conditions. Sufficiently large supercell (between 53 and 134 atoms) was constructed to ensure long range vibrations were accounted for. The multiplicity of the unit cell was determined by the ratio of the volume of the simulation supercell to the volume of a primitive cell. Corrections for basis-set superposition error and long-range dispersion interactions were included within PBEh-3c method ${ }^{46}$. This method is based upon the modified global hybrid functional PBEh, where percentage of Hartree-Fock exchange (42\%) is adjusted to get correct average bond length. Modified double- $\zeta$ Gaussian basis set dubbed def2-mSVP ${ }^{46,60}$ is defined within the method as well. The benchmark study, validating the accuracy of the chosen computational method is described in detail in Supporting Information.

Full geometry optimization, including both atom coordinates and unit cell parameters (a, b, $c, \alpha, \beta$, and $\gamma$ ), was applied to fully relax the geometry within the constraints of the space group symmetry. Threshold of convergence for crystal structure, based on the energy change between consecutive geometry optimization steps was set to $10^{-8}$ Bohr. Vibrational normal modes ${ }^{61}$ and IR intensities were calculated using the Berry phase method ${ }^{62}$. For the calculated vibrational frequencies, the scaling factor of 0.95 was applied for all of the spectral predictions ${ }^{46}$. The damping factor for the calculation of the Lorentzian linewidth at half maximum of absorption peak was set to $2.0 \mathrm{~cm}^{-1}$, in order to match the spectral shapes recorded at room temperature. Threshold on Self Consistent Field energy convergence was $10^{-10}$ Hartree. Truncation criteria for bielectronic integrals were as follows: overlap threshold for Coulomb integrals was $10^{-7}$, penetration threshold for Coulomb integrals was $10^{-7}$, overlap threshold for HF exchange integrals was $10^{-7}$, pseudo-overlap (HF exchange series) were $10^{-7}$ and $10^{-14}$. These criteria are based on well established guidelines which were described in $\operatorname{Ref}^{63}$.

\section{Results and discussion}

Geometry optimization, hydrogen bond, and charge distribution. Predicted unit cell parameters of fentanyl analogs are reported in Table S4. Their deviations from experimental values (collected in Table S3) are all within $0.3-0.9 \AA$ and $1.2-5.0^{\circ}$. The changes of unit cell volume upon optimization was less than $5 \%$ with the only exception of FOPFIZ, where this change was $~ 10 \%$.

The presence of $\mathrm{O}$ and $\mathrm{N}$ atoms in molecular structures of fentanyl and its analogs open the possibilities for the formation of hydrogen bonds (H-bonds). In fentanyl, TACDOS, and XALTAF, H-bond is formed by $\mathrm{N}$ atom of piperidine ring (N...H distances are $2.1,1.6$, and $2.1 \AA$, respectively). However, $\mathrm{O}$ and $\mathrm{N}$ atoms in 

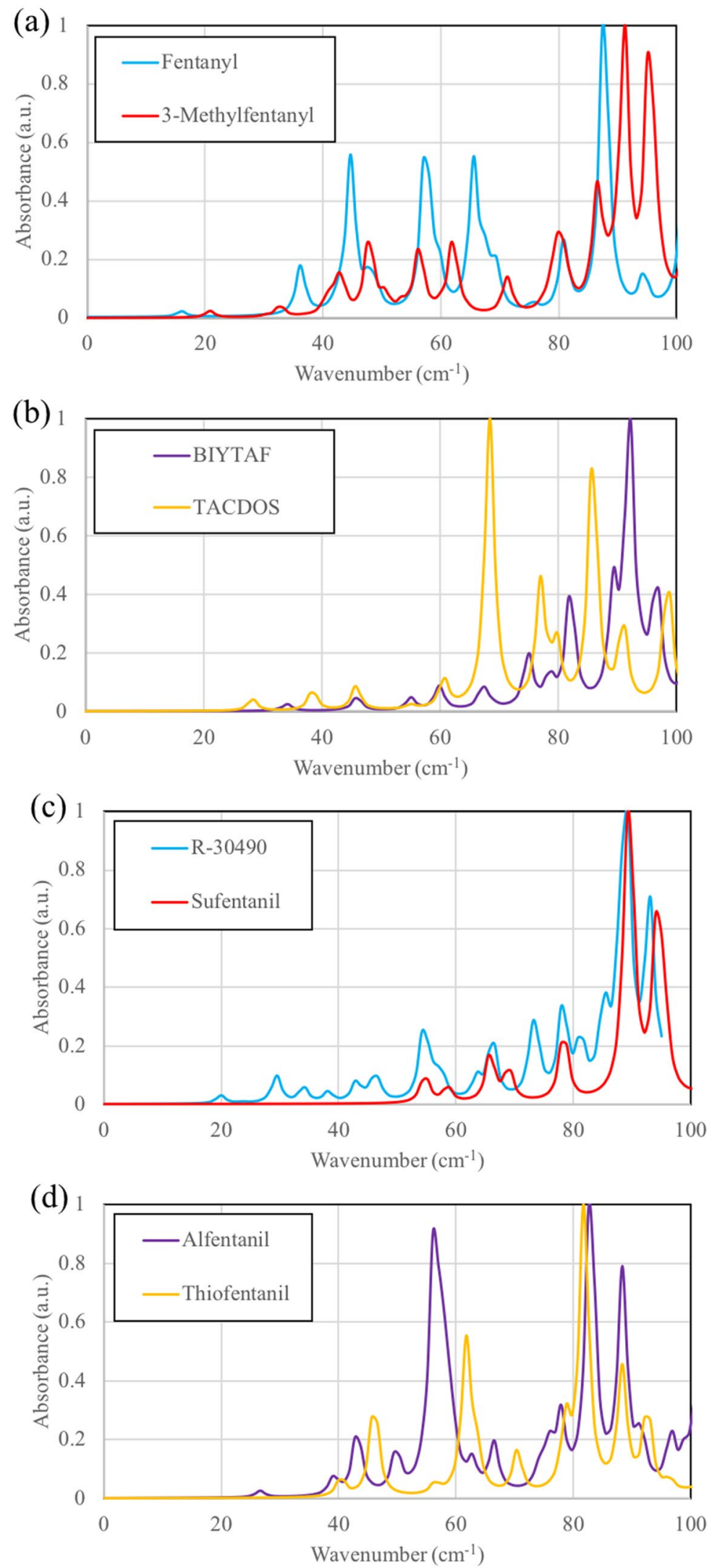

Figure 2. THz spectra of (a) fentanyl and 3-methylfentanyl, (b) BIYTAF and TACDOS, (c) R-30490 and sufentanil, and (d) alfentanil and thiofentanil. 
(a) Fentanyl
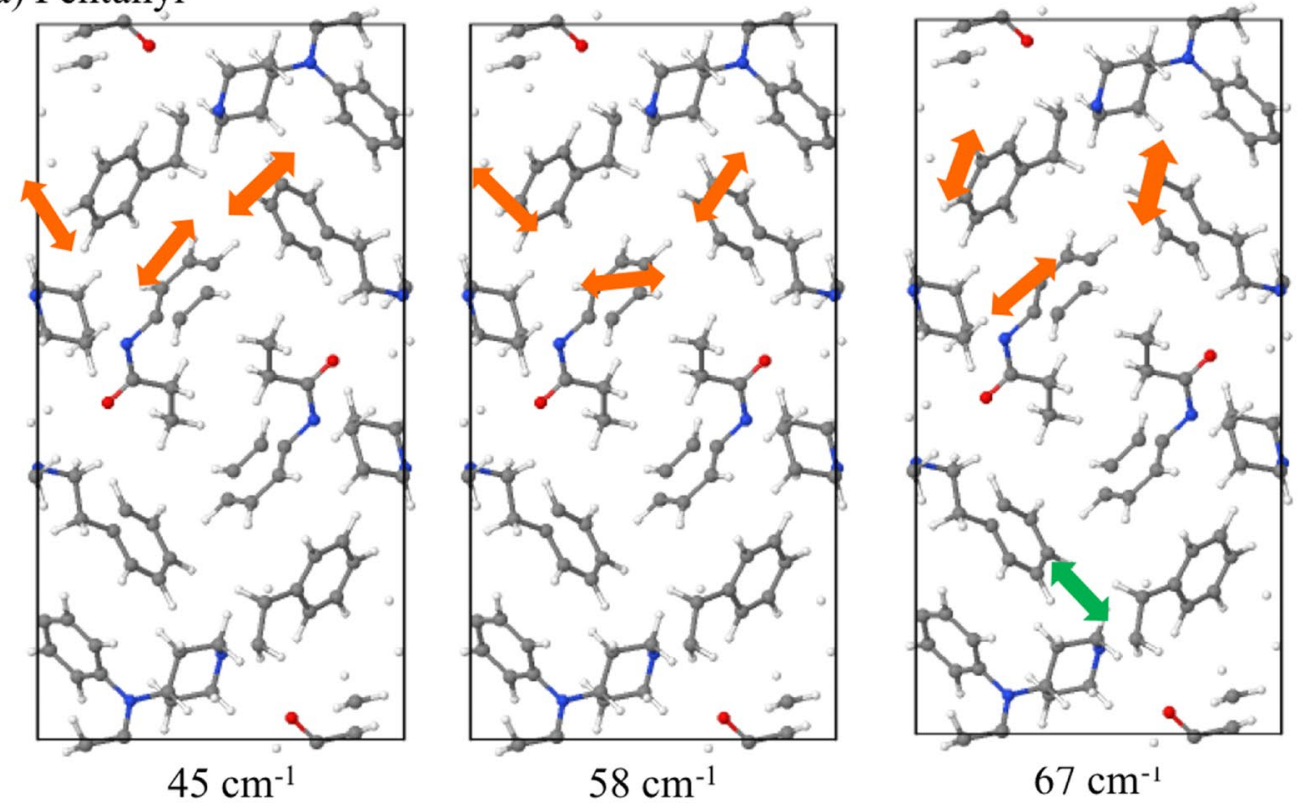

(b) 3-Methylfentanyl

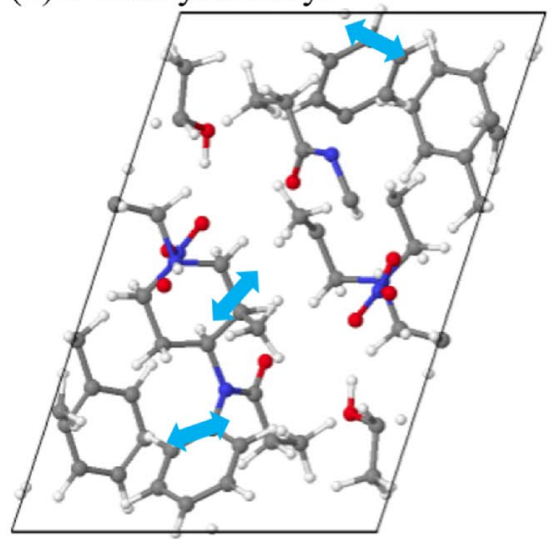

$48 \mathrm{~cm}^{-1}$

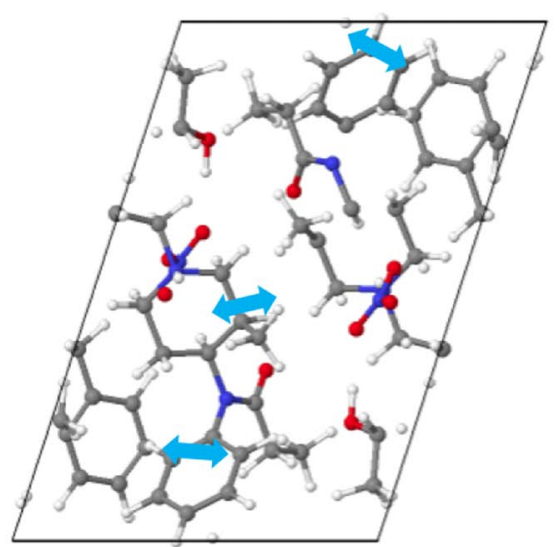

$57 \mathrm{~cm}^{-1}$

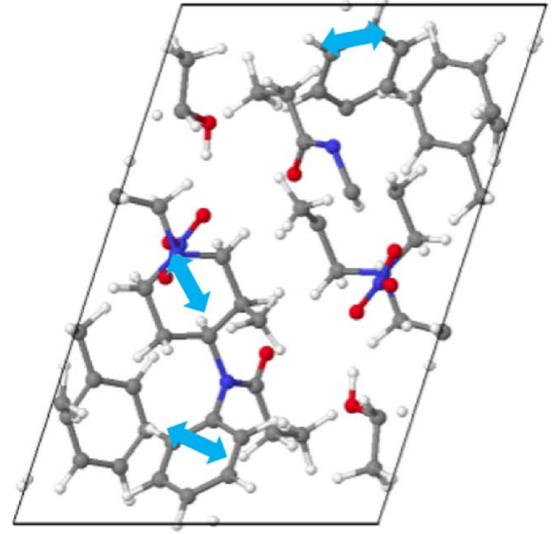

$62 \mathrm{~cm}^{-1}$

Figure 3. Normal modes of fentanyl in (a) 45, 58, and $67 \mathrm{~cm}^{-1}$ along with a-axis. Orange arrows show the vibrational direction of benzene and piperidine. Green arrow shows the formation of N...H H-bond. Normal modes of 3-methylfentanyl in (b) 48, 57, and $62 \mathrm{~cm}^{-1}$ along with b-axis. Blue arrows show the vibrational direction of benzene and 3-methylpiperidine. Carbon, hydrogen, nitrogen, and oxygen atoms are depicted as grey, white, blue, and red spheres, respectively.

$\mathrm{N}$-phenyl-propanamide do not form $\mathrm{H}$-bonds. Counterions in alfentanil and thiofentanil form $\mathrm{Cl}^{-} \ldots \mathrm{H}$ interactions $(\sim 2.1 \AA)$. Other sources of $\mathrm{H}$-bond interaction include solvent molecules water, oxalic acid, or hydrogen sulfate.

Hirshfeld population analysis ${ }^{64}$ describes molecular charge density in terms of atomic contribution and allows one to examine the nature of the charge transfer between various species in each crystal structure. The magnitude of Hirshfeld atomic charges is less dependent by basis set and is typically smaller than that of Mulliken charges ${ }^{65}$. Of all the fentanyl and its analogs, atomic charge of $\mathrm{O}$ atom in $\mathrm{N}$-phenyl-propanamide is $-0.555 \pm 0.016, \mathrm{~N}$ atom in $N$-phenyl-propanamide is $-0.363 \pm 0.047$, and $\mathrm{N}$ atom in piperidine ring is $-0.189 \pm 0.027$ (neutral) or $0.031 \pm 0.059$ (if protonated, $\mathrm{H}$ atom is $0.248 \pm 0.067$ ). Hence, the charge distribution in fentanyl and its analogs is consistent.

THz spectra of fentanyl and its analogs. Predicted $\mathrm{THz}$ absorption spectra of fentanyl and selected analogs are shown in Fig. 2. These correspond to polycrystalline samples (anisotropy is averaged out). Even one functional group difference results in a change of the crystal packing and thus the shape of absorption spectra. As one can see in Fig. 2, an additional methyl group for 3-methylfentanyl, a hydroxyl group for BIYTAF, and a carboxyl group for TACDOS results in different $\mathrm{THz}$ spectra. There are various medium peaks between 35 and 


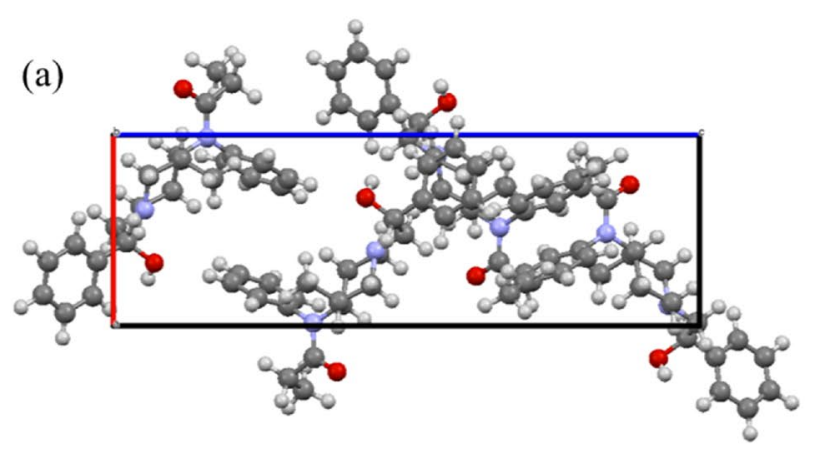

(b)

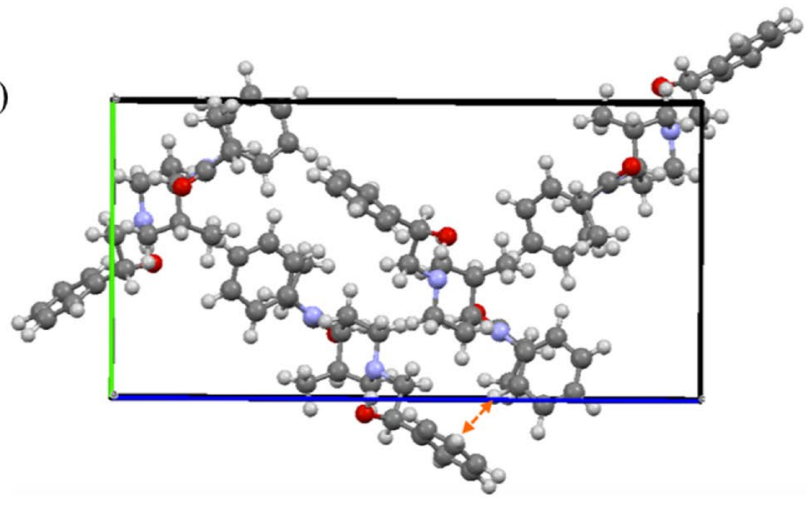

(c)

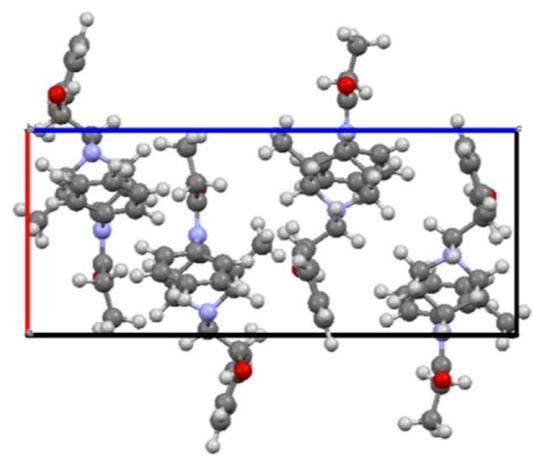

(d)

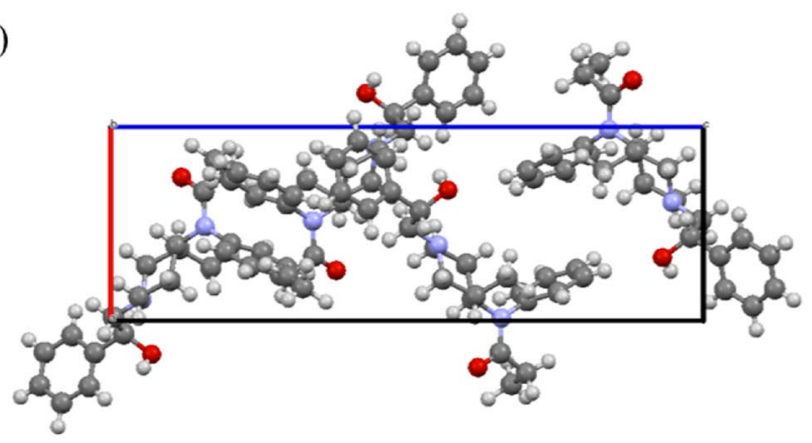

Figure 4. Crystal packing of ohmefentanyl stereoisomers (a) cis- $(2 R, 3 R, 4 S)$ viewed along with b-axis, (b) cis$(2 R, 3 R, 4 S)$ along with a-axis, (c) trans- $(2 S, 3 R, 4 R)$ along with b-axis, and (d) cis- $(2 S, 3 R, 4 R)$ viewed along with b-axis. Carbon, hydrogen, nitrogen, and oxygen atoms are depicted as grey, white, light purple, and red spheres, respectively.

$85 \mathrm{~cm}^{-1}$ of 3-methylfentanyl (Fig. 2a). Fentanyl, 3-methylfentanyl, and BIYTAF all have strongest peak above $85 \mathrm{~cm}^{-1}$. Normal mode visualization indicates that two peaks for fentanyl at 45 and $58 \mathrm{~cm}^{-1}$ arise from the intermolecular wiggling of T-shaped packing of the phenyl rings, which is not found in the other 3 analogs (Fig. 2a,b). The addition of methoxymethyl group on piperidine ring results in the appearance of several weak peaks in $\mathrm{THz}$ spectra of R-30490 (Fig. 2c) but no significant peaks like 45, 58, and $68 \mathrm{~cm}^{-1}$ in fentanyl and 68, 77, and $86 \mathrm{~cm}^{-1}$ in TACDOS (see Fig. 2a,b). Strong peaks arise from N...H H-bonds to piperidine ring in fentanyl and TACDOS. Comparison between TACDOS and TACDUY (with solvents water and methanol) is shown in Fig. S2. Medium to strong peaks between 25 and $75 \mathrm{~cm}^{-1}$ are from $\mathrm{H}$-bonds between solvents water and methanol. For alfentanil and thiofentanil (Fig. 2d), the strong peaks at 56 and $62 \mathrm{~cm}^{-1}$ arise from $\mathrm{Cl}$...H stretch between counterion and protonated piperidine ring. R4 group in both R-30490 and sufentanil (see Table 1) does not form strong $\mathrm{H}$-bonds, and dispersion interactions produce small but sharp peaks below $80 \mathrm{~cm}^{-1}$.

Figure 3a shows the vibrational normal modes of fentanyl at 45, 58, and $67 \mathrm{~cm}^{-1}$ and of 3-methylfentanyl at 48, 57, and $62 \mathrm{~cm}^{-1}$ are visualized in Fig. 3b. The difference in one methyl group leads to a distinct crystal packing and normal modes of fentanyl and 3-methylfentanyl. Similarly, sufentanil where the phenyl ring is substituted by thiophene ring results in weak peaks near 57,67 , and $78 \mathrm{~cm}^{-1}$, while alfentanil with the substitution to tetrazole ring leads to strong peaks at 56,85 , and $88 \mathrm{~cm}^{-1}$.

THz spectra of fentanyl analogs with chiral centers. The mode of molecular packing influences $\mathrm{THz}$ spectra significantly. Even for fentanyl analogs of the same atomic composition, stereoisomers induce changes in the packing and location of librational centers. Figure 4 shows the example of different crystal packing formed by the 3 ohmefentanyl isomers from R2, R3, and R4 groups (see Table 1) along with $b$ - or $a$-axis. Herringbone packing is visible when viewing cis- $(2 R, 3 R, 4 S)$ and cis- $(2 S, 3 R, 4 R)$ from 3 different directions in Fig. $4 \mathrm{a}, \mathrm{b}$, d. Orange arrow in Fig. $4 \mathrm{~b}$ shows T-shaped interaction. However, parallel packing is seen when viewing trans- $(2 S, 3 R, 4 R)$ along a- and b-axes while herringbone packing is seen along c-axis (Fig. 4c). The orientation of OH in R2 and $\mathrm{CH}_{3}$ in $\mathrm{R} 3$ groups influences the packing significantly.

$\mathrm{THz}$ absorption spectra of stereoisomers for selected fentanyl analogs are shown in Fig. 5. In ohmefentanyl (Fig. 5a) with stereoisomeric variations cis- $(2 R, 3 R, 4 S)$ and cis- $(2 S, 3 R, 4 R)$, similar THz spectra shows both the crystal packing include T-shaped interaction (Fig. 4a,d). There is merely a slight wavelength shift and it represents the packing dominates $\mathrm{THz}$ spectra. Intermolecular $\mathrm{H}$-bond between ohmefentanyl molecules is absent even in the presence of $\mathrm{OH}$ group in R2 (see Table 1). Conversely, ohmefentanyl trans- $(2 S, 3 R, 4 S)$ exhibits a different spectrum with the peak at $79 \mathrm{~cm}^{-1}$ because of $\pi-\pi$ stacking interaction from phenyl ring in $\mathrm{R} 1$, which is absent in $c i s-(2 R, 3 R, 4 S)$ and $c i s-(2 S, 3 R, 4 R)$. 

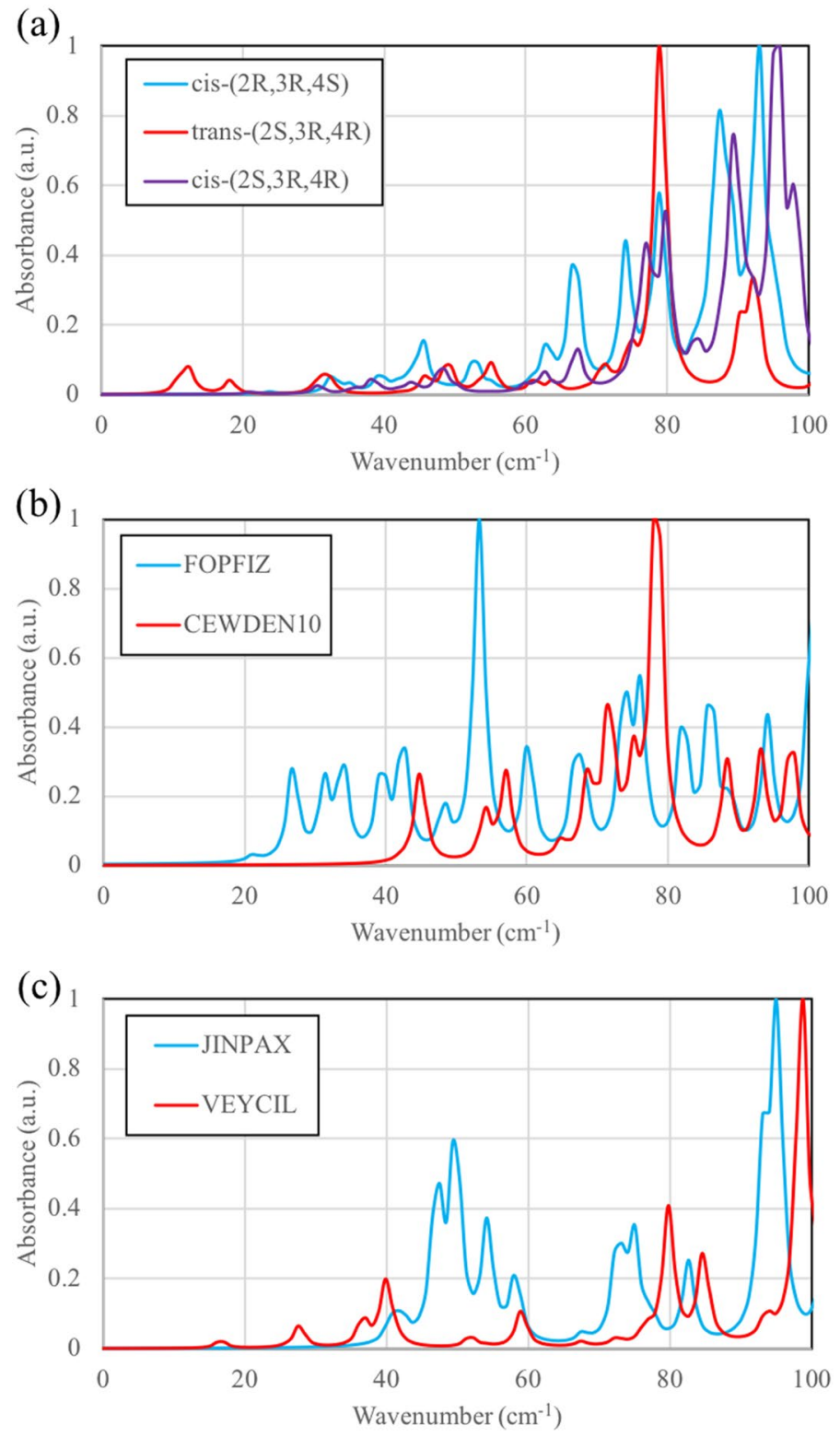

Figure 5. THz spectra of stereoisomers (a) ohmefentanyl (cis- $(2 R, 3 R, 4 S)$, trans- $(2 S, 3 R, 4 S)$, and cis- $(2 S, 3 R, 4 R))$, (b) FOPFIZ (trans) and CEWDEN10 (cis), and (c) JINPAX $(2 R, 5 S)$ and VEYCIL $(2 S, 5 S)$.

Comparison between FOPFIZ (trans) and CEWDEN10 (cis) is presented in Fig. 5b. Both of them have several small peaks with one strong peak at 53 or $78 \mathrm{~cm}^{-1}$. Because of the solvent molecules and/or counterion in FOPFIZ and CEWDEN10, small and medium peaks are present to reflect the formation of multiple $\mathrm{H}$-bonds. The strongest peak of FOPFIZ at $53 \mathrm{~cm}^{-1}$ shows the T-shaped phenyl ring interactions (see Fig. $6 \mathrm{a}$ ). The strongest peak of CEWDEN10 at $78 \mathrm{~cm}^{-1}$ shows interactions between the dangling ethyl groups instead of phenyl rings $\left(\mathrm{CH}_{3} \ldots \pi\right.$ interaction, Fig. 6b).

JINPAX $(2 R, 5 S)$ and VEYCIL $(2 S, 5 S)$ in Fig. 5 c have similar situation that combine several small peaks with one or two strong peaks at 95 or $98 \mathrm{~cm}^{-1}$ (shown in Fig. $6 \mathrm{c}, \mathrm{d}$ ). THz spectra therefore distinguishes stereoisomers in these examples. JINPAX contains hydrogen sulfate that can form $\mathrm{H}$-bond with nitrogen atom in piperidine ring. $\mathrm{CH} . . . \pi$ and parallel/T-shaped phenyl ring interactions determines the appearance of medium and strong peaks on both JINPAX and VEYCIL spectra.

All the other THz spectra of fentanyl analogs FBPIPA, BEGZUJ, IFIGIN, ZIZPON, and XALTAF can be found in Fig. S3. Strongest peaks above $75 \mathrm{~cm}^{-1}$ are based upon parallel $\pi-\pi$ stacking, and T-shaped CH... $\pi$, $\mathrm{CH}_{3} \ldots \pi$ interactions. Note that there is no significant $\mathrm{H}$-bond interaction in these analogs. Medium and small peaks below $75 \mathrm{~cm}^{-1}$ arise from long-distance, displaced stacking $\pi-\pi$ and T-shaped interactions or from solvent molecules and counterions. 
(a)

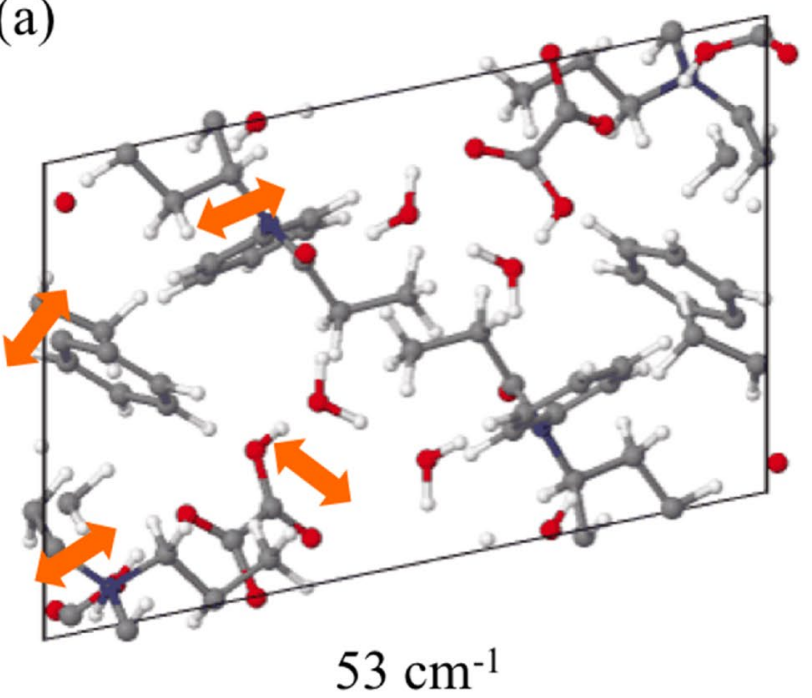

(c)

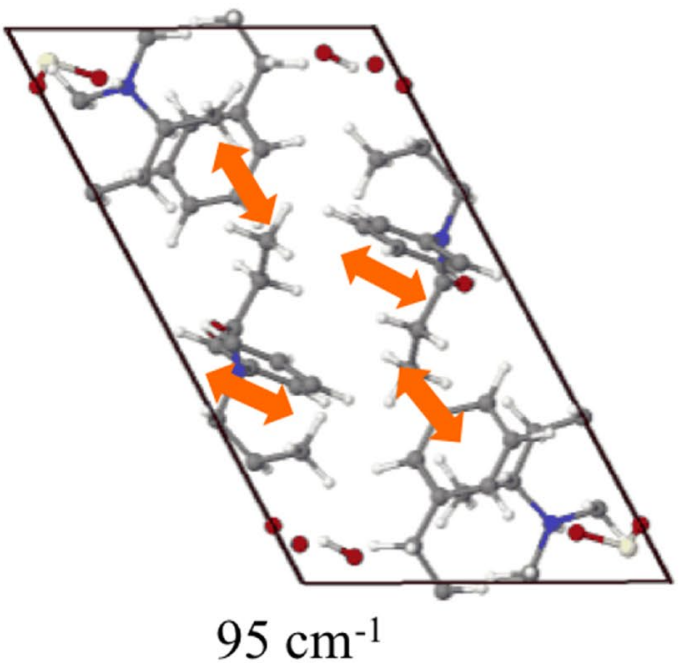

(b)

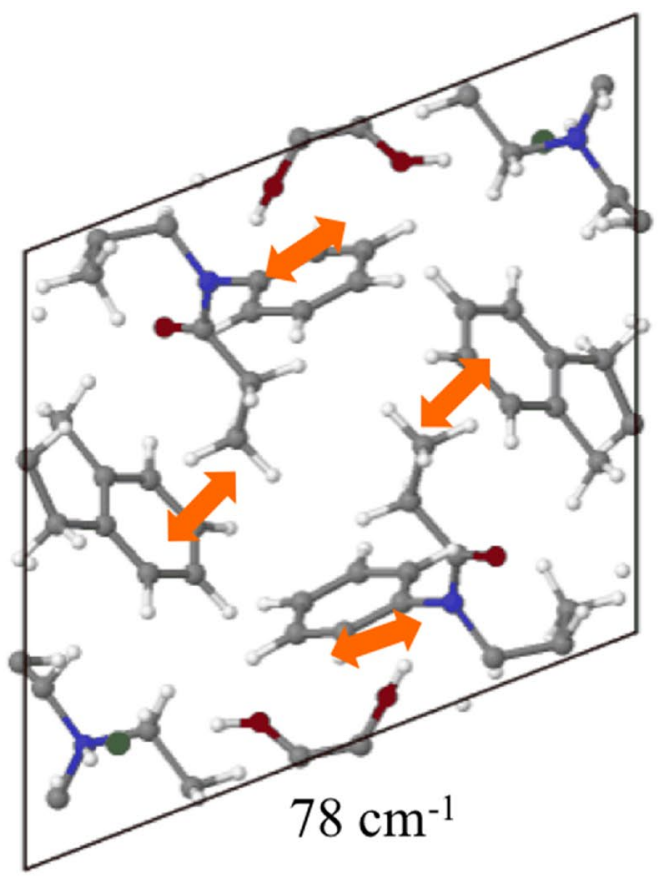

(d)

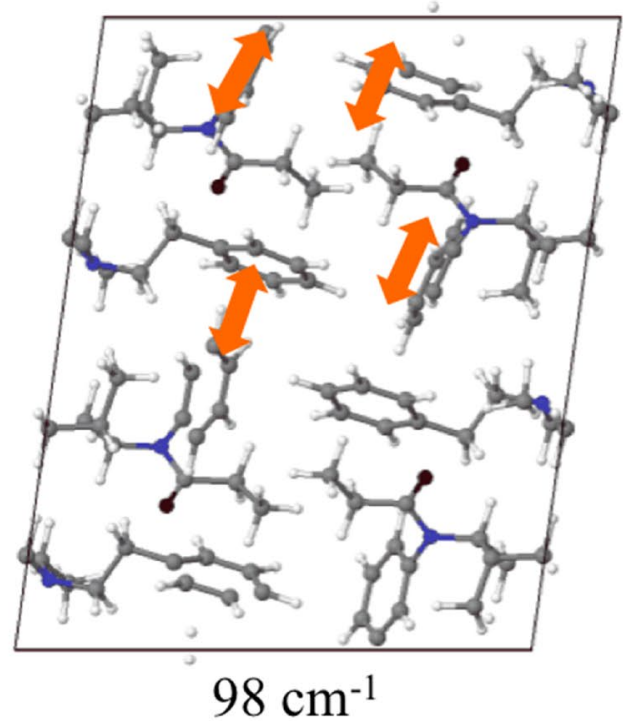

Figure 6. Normal modes of (a) FOPFIZ in $53 \mathrm{~cm}^{-1}$ along with a-axis, (b) CEWDEN10 in $78 \mathrm{~cm}^{-1}$ along with a-axis, (c) JINPAX in $95 \mathrm{~cm}^{-1}$ along with c-axis, and (d) VEYCIL in $98 \mathrm{~cm}^{-1}$ along with b-axis. Strongest peaks in $\mathrm{THz}$ range are shown. Carbon, hydrogen, nitrogen, and oxygen atoms are depicted as grey, white, blue, and red spheres, respectively.

\section{Conclusions}

Spectra of fentanyl and its analogs in THz range have specific characteristics. First, the generic picture of $\mathrm{THz}$ spectra of fentanyl and its analogs is that between $75-100 \mathrm{~cm}^{-1}$, there are 2-4 strong peaks from significant $\pi-\pi$ interactions and/or H-bonds. Second, THz range between $20-75 \mathrm{~cm}^{-1}$ consists of small to medium peaks because of non-ideal $\pi-\pi$ interactions, H-bond, and dispersion interaction. Third, the existence of $\pi-\pi$ interactions or $\mathrm{H}$-bonds that belong to solvent molecules or counterions leads to medium or strong peaks between $20-75 \mathrm{~cm}^{-1}$. Compared with compounds that are in white powder form (see Fig. S1), THz spectra of fentanyl and its analogs have the preference of peaks at certain $\mathrm{THz}$ range. The three characteristics may help to apply ML to predict unknown street drugs in the future and enable the detector design for synthetic opioids. 


\section{References}

1. Ogawa, N., Nagase, H., Endo, T., Loftsson, T. \& Ueda, H. Crystal structure of fentanyl base. X-Ray Struct. Anal. Online 25, 83-84 (2009).

2. Uğur, B., Oğurlu, M., Yılmaz, S. \& Kıvrak, V. Determining the optimal fentanyl dose for dilation and curettage procedures. Clin. Exp. Obstet. Gynecol. 39, 509-511 (2012).

3. Labroo, R. B., Paine, M. F., Thummel, K. E. \& Kharasch, E. D. Fentanyl metabolism by human hepatic and intestinal cytochrome P450 3A4: Implications for interindividual variability in disposition, efficacy, and drug interactions. Drug Metab. Dispos. 25, 1072-1080 (1997).

4. Armenian, P., Vo, K. T., Barr-Walker, J. \& Lynch, K. L. Fentanyl, fentanyl analogs and novel synthetic opioids: A comprehensive review. Neuropharmocology 134, 121-132 (2018).

5. Stanley, T. H., Egan, T. D. \& Van Aken, H. A. Tribute to Dr. Paul A. J. Janssen: Entrepreneur extraordinaire, innovative scientist, and significant contributor to anesthesiology. Anesth Analg 106, 451-462 (2008).

6. Peeters, O. M., Blaton, N. M. \& De Ranter, C. J. N-\{4-(Methoxymethyl)-1-[2-(2-thienyl)ethyl]-4-piperidinyl\}-N-phenylpropanamide: Sufentanil. Acta Crystallogr. Sect. B 35, 999-1001 (1979).

7. Durant, F., Michel, A., Lebrun, B. \& Evrard, G. X-ray crystal structure of alfentanyl (R39209), an analgesic of the fentanyl family. Bull. Soc. Chim. Belg. 96, 331-332 (1987).

8. Bürkle, H., Dunbar, S. \& Van Aken, H. Remifentanil: A Novel, Short-Acting, $\mu$-opioid. Anesth. Analg. 83, 646-651 (1996).

9. Tateishi, T. et al. Identification of human liver cytochrome P-450 $3 \mathrm{~A} 4$ as the enzyme responsible for fentanyl and sufentanil N-dealkylation. Anesth. Analg. 82, 167-172 (1996).

10. Poklis, A. \& Backer, R. Urine concentrations of fentanyl and norfentanyl during application of Duragesic ${ }^{\circledR}$ transdermal patches. J. Anal. Toxicol. 28, 422-425 (2004).

11. Kuhlman, J. J. Jr., McCaulley, R., Valouch, T. J. \& Behonick, G. S. Fentanyl use, misuse, and abuse: a summary of 23 postmortem cases. J. Anal. Toxicol. 27, 499-504 (2003).

12. Faust, A. C., Terpolilli, R. \& Hughes, D. W. Management of an oral ingestion of transdermal fentanyl patches: a case report and literature review. Case Rep. Med. https://doi.org/10.1155/2011/495938 (2011).

13. Pergolizzi Jr, J. V., LeQuang, J. A., Taylor Jr, R., Raffa, R. B. \& Group, N. R. Going beyond prescription pain relievers to understand the opioid epidemic: The role of illicit fentanyl, new psychoactive substances, and street heroin. Postgrad. Med. 130, 1-8 (2018).

14. Wax, P. M., Becker, C. E. \& Curry, S. C. Unexpected, "gas" casualties in Moscow: A medical toxicology perspective. Ann. Emerg. Med. 41, 700-705 (2003).

15. Bucerius, S. M. \& Haggerty, K. D. Fentanyl behind bars: The implications of synthetic opiates on prisoners and correctional officers. Int. J. Drug Policy 71, 133-138 (2019).

16. Forbes, T. P., Lawrence, J., Verkouteren, J. R. \& Verkouteren, R. M. Discriminative potential of ion mobility spectrometry for the detection of fentanyl and fentanyl analogues relative to confounding environmental interferents. Analyst 144, 6391-6403 (2019).

17. Verkouteren, J. R., Lawrence, J., Verkouteren, R. M. \& Sisco, E. Method for evaluating ion mobility spectrometers for trace detection of fentanyl and fentanyl-related substances. Anal. Methods 11, 6043-6052 (2019).

18. Ohta, H., Suzuki, S. \& Ogasawara, K. Studies on fentanyl and related compounds IV. Chromatographic and spectrometric discrimination of fentanyl and its derivatives. J. Anal. Toxicol. 23, 280-285 (1999).

19. Mao, C.-L. et al. Development of an enzyme-linked immunosorbent assay for fentanyl and applications of fentanyl antibody-coated nanoparticles for sample preparation. J. Pharm. Biomed. Anal. 41, 1332-1341 (2006).

20. Schüttler, J. \& White, P. F. Optimization of the radioimmunoassays for measuring fentanyl and alfentanil in human serum. Anesthesiology 61, 315-320 (1984).

21. Sisco, E., Verkouteren, J., Staymates, J. \& Lawrence, J. Rapid detection of fentanyl, fentanyl analogues, and opioids for on-site or laboratory based drug seizure screening using thermal desorption DART-MS and ion mobility spectrometry. Foren. Chem. 4, 108-115 (2017).

22. Haddad, A., Comanescu, M. A., Green, O., Kubic, T. A. \& Lombardi, J. R. Detection and quantitation of trace fentanyl in heroin by surface-enhanced raman spectroscopy. Anal. Chem. 90, 12678-12685 (2018).

23. Salemmilani, R., Moskovits, M. \& Meinhart, C. D. Microfluidc analysis of fentanyl-laced heroin samples by surface-enhanced Raman spectroscopy in a hydrophobic medium. Analyst 144, 3080-3087 (2019).

24. Ahmar, H., Fakhari, A. R., Tabani, H. \& Shahsavani, A. Optimization of electromembrane extraction combined with differential pulse voltammetry using modified screen-printed electrode for the determination of sufentanil. Electrochim. Acta 96, 117-123 (2013).

25. Neu, J. \& Schmuttenmaer, C. A. Tutorial: An introduction to terahertz time domain spectroscopy (THz-TDS). J. Appl. Phys. 124, $231101(2018)$

26. Mittleman, D. M. Perspective: Terahertz science and technology. J. Appl. Phys. 122, 230901 (2017).

27. Lu, M. et al. Detection and identification of illicit drugs using terahertz imaging. J. Appl. Phys. 100, 103104 (2006).

28. Davies, A. G., Burnett, A. D., Fan, W., Linfield, E. H. \& Cunningham, J. E. Terahertz spectroscopy of explosives and drugs. Mater. Today 11, 18-26 (2008).

29. Flippen-Anderson, J. L., George, C., Bertha, C. M. \& Rice, K. C. X-ray crystal structures of potent receptor ligands: Etonitazene, cis-(+)-3-methylfentanyl, etorphine, diprenorphine, and buprenorphine. Heterocycles 39, 751-766 (1994).

30. Wang, Z.-X. et al. Stereoisomers of N-[1-(2-Hydroxy-2-phenylethyl)-3-methyl-4-piperidyl]-N-phenylpropanamide: Synthesis, stereochemistry, analgesic activity, and opioid receptor binding characteristics. J. Med. Chem. 38, 3652-3659 (1995).

31. Brine, G. A. et al. Enantiomers of diastereomeric cis-N-[1-(2-Hydroxy-2-phenylethyl)-3-methyl-4-piperidyl]-N-phenylpropanamides: synthesis, X-ray analysis, and biological activities. J. Med. Chem. 38, 1547-1557 (1995).

32. Michel, A., Lebrun, B., Evrard, G. \& Durant, F. Structure of N-[1-(2-Hydroxy-2-phenylethyl)-4-piperidyl]-N-phenylpropanamide Hydrochloride (R4333*), an analgesic of the fentanyl family. Acta Crystallogr. Sect. B 38, 2961-2963 (1982).

33. Nichol, G. S., Kumirov, V. K., Vardanyan, R. \& Hruby, V. J. Proton sharing and transfer in some zwitterionic compounds based on 4-oxo-4-((1-phenethylpiperidin-4-yl)(phenyl)amino)alconoic acids. CrysEngComm 12, 3651-3657 (2010).

34. Karapetyan, A. A., Struchkov, Y. T., Martirosyan, V. O., Vartanyan, R. S. \& Vartanyan, S. A. The structure of 4-anilinopiperidine analgesics. III. The structure of the acid sulfate of 1(e)-(2-phenethyl)-2(e),5(e)-dimethyl-4(e)-(N-propylylanilino)piperidine. J. Struct. Chem. 31, 307-311 (1990).

35. Karapetyan, A. A. et al. Structure and Activity of stereoisomers of fenaridine. Pharm. Chem. J. 23, 387-394 (1989).

36. Klein, C. L., Stevens, E. D., Fifer, E. K. \& Borne, R. F. Molecular structure of two conformationally restrained fentanyl analogues: cis- and trans-isomers of N-\{3-Methyl-1-[2-(1,2,3,4-tetrahydro)naphthyl]-4-piperidinyl $\}$-N-phenylpropanamide. J. Pharm. Sci. 74, 1147-1151 (1985).

37. Koch, M. J. H. \& Evrard, G. N-\{1-[3-(p-Fluorobenzoyl)propyl]-4-piperidyl\}propionanilide. Acta Crystallogr. Sect. B 30, 237-239 (1974).

38. Jimeno, M. L. et al. Fentanyl and its analogue N-(1-phenylpyrazol-3-yl)-N-[1-(2-phenylethyl)-4-piperidyl]propanamide: 1H- and 13C-NMR spectroscopy, X-ray crystallography, and theoretical calculations. Chem. Pharm. Bull. 51, 929-934 (2003). 
39. Deschamps, J. R., George, C. \& Flippen-Anderson, J. L. (1R)-2-[(3R,4S)-3-Methyl-4-(N-phenyl-N-propionylamino)piperidin-1yl]-1-phenylethyl p-bromobenzoate and $\mathrm{N}-\{(3 \mathrm{R}, 4 \mathrm{~S})-1-[(2 \mathrm{~S})-2$-(4-bromophenyl)-2-hydroxyethyl]-3-methyl-piperidin-4-yl\}-Nphenylacrylamide. Acta Crystallogr. Sect. C 58, o362-o364 (2002).

40. Erman, L. Y., Mindrul, V. F., Khromova, N. Y. \& Kurochkin, V. K. Molecular structure of 1(phenethyl)-4(3-trimethyl germyl) propioanilidopiperidine hydrochloride. J. Struct. Chem. 36, 520-524 (1995).

41. Mitsuya, M., Kawakami, K., Ogino, Y., Miura, K. \& Mase, T. Stereoselective synthesis of a new muscarinic M3 receptor antagonist, J-104129. Bioorg. Med. Chem. Lett. 9, 2037-2038 (1999).

42. Ferraro, J. \& Walker, A. Comparison of the infrared spectra $(4000-70 \mathrm{~cm}-1)$ of several hydrated and anhydrous salts of transition metals. J. Chem. Phys. 42, 1278-1285 (1965).

43. Koch, M. J. H., De Ranter, C. J., Rolies, M. \& Dideberg, O. N-(4-(Methoxymethyl)-1-(2-phenylethyl)-4-piperidinyl)-N-phenylpropanamide. Acta Crystallogr. Sect. B 32, 2529-2531 (1976).

44. Hu, J., Zhang, J., Zhu, G. \& Li, Y. Crystal structure of the methyl-4-[N-(1-oxypropyl)-N-phenylamino]-1-[2-(2-thienyl)ethyl]4-piperidinecarboxylate (R31826) hydrochloride. Chin. J. Struct. Chem. 8, 91 (1989).

45. Takahashi, M. Terahertz vibrations and hydrogen-bonded networks in crystals. Crystals 4, 74-103 (2014).

46. Grimme, S., Brandenburg, J. G., Bannwarth, C. \& Hansen, A. Consistent structures and interactions by density functional theory with small atomic orbital basis sets. J. Chem. Phys. 143, 054107 (2015).

47. Perdew, J. P., Burke, K. \& Ernzerhof, M. Generalized gradient approximation made simple. Phys. Rev. Lett. 77, 3865-3868 (1996).

48. Lipiński, P. F. J., Jarończyk, M., Ostrowski, S., Dobrowolski, J. C. \& Sadlej, J. Conformation of six fentanyls revisited. Comput. Theor. Chem. 1086, 18-24 (2016).

49. Jiang, H. L. et al. Theoretical studies on opioid receptors and ligands. I. Molecular modeling and QSAR studies on the interaction mechanism of fentanyl analogs binding to $\mu$-opioid receptor. Int. J. Quantum Chem. 78, 285-293 (2000).

50. Lipiński, P. F. J., Jarończyk, M., Dobrowolski, J. C. \& Sadlej, J. Molecular dynamics of fentanyl bound to $\mu$-opioid receptor. J. Mol. Model. 25, 144-160 (2019).

51. Jaime, C., Virgili, A., Portillo, S., Caparrós, A. \& Burusco, K. K. Configuration and conformation of alfentanil hydrochloride. Conformational study by NMR and theoretical calculations. Magn. Reson. Chem. 52, 440-447 (2014).

52. Groom, C. R., Bruno, I. J., Lightfoot, M. P. \& Ward, S. C. The Cambridge structural database. Acta Crystallogr. Sect. B 72, 171-179 (2016).

53. Björkman, T. CIF2Cell: Generating geometries for electronic structure programs. Comput. Phys. Commun. 182, 1183-1186 (2011).

54. Schaftenaar, G. \& Noordik, J. H. Molden: a pre- and post-processing program for molecular and electronic structures. J. Comput. Aided Mol. Des. 14, 123-134 (2000).

55. https://chemaxon.com (accessed December 9, 2020).

56. Macrae, C. F. et al. Mercury 4.0: From visualization to analysis, design and prediction. J. Appl. Crystallogr. 53, 226-235 (2020).

57. http://crysplot.crystalsolutions.eu/ (accessed December 9, 2020).

58. https://office.microsoft.com/excel (accessed December 9, 2020).

59. Dovesi, R. et al. Quantum-mechanical condensed matter simulations with CRYSTAL. WIREs Comput. Mol. Sci. 8, e1360 (2018).

60. Schäfer, A., Horn, H. \& Alhrichs, R. Fully optimized contracted Gaussian basis sets for atoms Li to Kr. J. Chem. Phys. 97, 2571-2577 (1992).

61. Pascale, F. et al. The calculation of the vibrational frequencies of crystalline compounds and its implementation in the CRYSTAL code. J. Comput. Chem. 25, 888-897 (2004).

62. Noel, Y., Zicovich-Wilson, C. M., Civalleri, B., D’Arco, P. \& Dovesi, R. Polarization properties of ZnO and BeO: An ab initio study through the Berry phase and Wannier functions approaches. Phys. Rev. B 65, 014111 (2001).

63. Erba, A., Baima, J., Bush, I., Orlando, R. \& Dovesi, R. Large-scale condensed matter DFT simulations: performance and capabilities of the CRYSTAL code. J. Chem. Theory Comput. 13, 5019-5027 (2017).

64. Hirshfeld, F. L. Bonded-atom fragments for describing molecular charge densities. Ther. Chim. Acta 44, 129-138 (1977).

65. Mulliken, R. J. Electronic population analysis on LCAO-MO molecular wave functions. I.. J. Chem. Phys. 23, 1833-1840 (1955).

\section{Acknowledgements}

The project or effort depicted was or is sponsored by the Defense Advanced Research Projects Agency (DARPA) Agreement Number: D18AP00040. The readers should note that the content of the information does not necessarily reflect the position or the policy of the Government, and no official endorsement should be inferred. This research used resources Cori of the National Energy Research Scientific Computing Center (NERSC), a U.S. Department of Energy Office of Science User Facility operated under Contract No. DE-AC02-05CH11231, and resources Stampede2 of the Extreme Science and Engineering Discovery Environment (XSEDE), which is supported by National Science Foundation grant number ACI-1548562. A.E.M. is grateful for partial support of this work by the "Improving of the Competitiveness" program of the National Nuclear Research University "MEPhI" and by the Act 211 of the Government of the Russian Federation (Contract No. 02.A03.21.0011). The authors also acknowledge the University of Central Florida Advanced Research Computing Center (https://arcc.ist.ucf. edu) for providing computational resources and support.

\section{Author contributions}

C.-H.W., A.C.T., A.E.M., and S.S.V. designed research. C.-H.W., A.E.M., A.C.T., M.X. and S.S.V. performed research, and C.-H.W., A.E.M., and S.S.V. wrote the manuscript.

\section{Competing interests}

The authors declare no competing interests.

\section{Additional information}

Supplementary Information The online version contains supplementary material available at https://doi. org/10.1038/s41598-021-83536-y.

Correspondence and requests for materials should be addressed to A.E.M.

Reprints and permissions information is available at www.nature.com/reprints.

Publisher's note Springer Nature remains neutral with regard to jurisdictional claims in published maps and institutional affiliations. 
(c) (i) Open Access This article is licensed under a Creative Commons Attribution 4.0 International cc) License, which permits use, sharing, adaptation, distribution and reproduction in any medium or format, as long as you give appropriate credit to the original author(s) and the source, provide a link to the Creative Commons licence, and indicate if changes were made. The images or other third party material in this article are included in the article's Creative Commons licence, unless indicated otherwise in a credit line to the material. If material is not included in the article's Creative Commons licence and your intended use is not permitted by statutory regulation or exceeds the permitted use, you will need to obtain permission directly from the copyright holder. To view a copy of this licence, visit http://creativecommons.org/licenses/by/4.0/.

(C) The Author(s) 2021 